\title{
The pathogenesis and treatment of primary liver cancer
}

\author{
Chu Tianjiao \\ Institute of product quality supervision and inspection, Nantong 226000,China
}

Keywords: Primary liver cancer, pathogenesis, specific treatment

\begin{abstract}
The occurrence of liver cancer seriously affects the life and health of the human body. Each year, around one million people around the world are diagnosed with liver cancer. China is a high incidence area of liver cancer. Clinical data show that in recent years, the number of patients with liver cancer has increased and the trend of age is low. Related data show that in urban areas, the fatality rate of HCC is second only to lung cancer, and second only to gastric cancer in rural areas. Liver cancer has become a major killer of human health. It seriously threatens human life and health. How to treat liver cancer effectively and quickly becomes one of the most urgent tasks for the basic researchers.
\end{abstract}

\section{Liver cancer}

\section{1 kinds of liver cancer}

Liver cancer, broadly speaking, includes primary liver cancer and metastatic liver cancer. People usually refer to liver cancer, mainly refers to primary liver cancer. In narrow sense, HCC can be divided into hepatocellular carcinoma, cholangiocarcinoma and mixed hepatocellular carcinoma according to cell types. Therefore, according to the different pathogenesis of HCC, liver cancer is divided into five types: primary liver cancer, hepatocellular carcinoma, cholangiocarcinoma, [1], metastatic liver cancer and secondary liver cancer. The pathogenesis and current treatment of primary hepatocellular carcinoma (HCC) are reviewed in this paper.

\section{2 the pathogenesis of primary liver cancer}

Including the main predisposing risk factors for primary liver cancer: hepatitis B virus, hepatitis C virus and aflatoxin contaminated food, familial genetic factors, drinking water pollution source pollution, smoking and drinking and other external factors [8]. Among the many carcinogenic factors, the main roles are hepatitis history, hepatitis B virus infection, hepatitis C virus infection, and family history of liver cancer.

From the perspective of molecular biology, primary liver cancer is a healthy life in normal cells are transformed into variation, uncontrolled cell proliferation and malignant tumor invasive and complicated pathological changes. From the point of view of cytokine regulation, the formation of malignant tumor cells is a multi-step, complex, long and evolving process, which is involved in the regulation and expression of many genes. This process involves interactions among a variety of cytokines, [4]. From the molecular point of view, the occurrence of primary liver cancer is the final result of the imbalance of homeostatic mechanism caused by the balance of the positive and negative feedback and the bidirectional regulation mechanism. The genes that regulate homeostasis include proto oncogene and tumor suppressor gene two, [2]. The occurrence of primary liver cancer is the final result of the combined action of proto oncogene activation and abnormal expression of tumor suppressor genes. The oncogene is known as proto oncogene, a toxic substance that causes the proto oncogene to change. At the level of nucleic acid, it is usually expressed as point mutation, virus induction and promoter insertion, gene amplification, chromosome breakage and rearrangement, etc. [4-5].

\subsubsection{The pathogenesis of primary hepatocellular carcinoma caused by point mutation}

[2-3] in the pathogenesis of point mutations in the ras gene: the most common is activated, resulting in twelfth codon nucleotide sequences of long chain by the mutation of GGT to GTT, the 
original codon corresponding encoding RAS protein. The protein plays an important role in regulating cell growth, differentiation and so on. The common structure of RAS protein is two kinds of common forms of Ras-GTP active state and Ras-GDP inactive state. Point mutation causes GAP not to act on the structure of Ras-GTP protein, but is persistent in the activation state, thus transmitting the proliferating signal to the nucleus, resulting in malignant transformation of cells. A protein encoded by a tumor suppressor gene that blocks the formation of tumor blocks. When a point mutation causes the gene to fail to express normally or its product is inactivated, the cell becomes cancerous. During the regulation of Ras gene, the cytokines regulated include RB, P21, P53 and so on. They also include known downstream target genes such as GADD45, MDM2, BAX, IGF-bp3, and PIG3.

\subsubsection{Pathogenesis of primary liver cancer caused by hepatitis $B$ virus}

Hepatitis B virus (HBV) [6], a globular eosinophilic DNA virus that includes protein capsid and single stranded DNA molecules, and DNA polymerase. HBV enters the cell through the cell membrane, and the DNA that takes off the protein capsid enters the nucleus through a nuclear pore, forming a covalently closed ring of DNA molecules (cccDNA), or integrates into the genome of the cell to form recombinant DNA. After that, it is transcribed into several RNA to synthesize hepatitis B surface, hepatitis B core, or polymerase. The carcinogenic mechanism of HBV can be divided into trans activation and CIS activation. CIS activation, that is, the insertion of HBV-DNA nucleotide sequences into the liver cell proto oncogene, activates or enhances the expression of oncogenes by acting with promoters. Trans activation is the HBV-DNA nucleotide sequence that is randomly integrated into the stem cell genome DNA, which translates into proteins after transcription and activates its own genes or proto oncogenes. It is now generally thought that the trans activator may be HBxAg (hepatitis B virus X antigen).

\subsubsection{Pathogenesis of primary hepatocellular carcinoma caused by aflatoxin}

Among the more than 20 aflatoxins, the most potent cause of aflatoxin is aflatoxin B1[6]. Who exist in aflatoxin moldy peanuts, beans and other cereal crops. It is an important risk factor for mutations in codon 249th causing P53. Usually, exposure to aflatoxin B1 (AFB1), can lead to p53 gene 249th codon third mutations (nucleotide substitution from $\mathrm{G}$ to $\mathrm{T}$ ), so that the amino acid codon encoding by serine (Ser) into arginine (Arg). In addition, the p53 gene also has many codons (226, 243, 244, 245, 248) mutations, introns mutations, and introns and exons splice site mutations, etc.. Depending on the nature of the mutation, p53 protein could not express or express instability.

1.2.4 signaling pathway is involved in the pathogenesis of primary hepatocellular carcinoma

The aberrant activation of multiple signaling pathways is also involved in the initiation and evolution of HCC, [7]. Growth factors are signaling molecules that play a key role in cell proliferation, including epithelial growth factor (EGF), hepatocyte growth factor (HGF), platelet derived endothelial growth factor, and so on. These growth factors (known as ligand) after specific binding with the receptor, activation of Ras/MAPK signaling pathway through the activation of receptor tyrosine kinase activity, and ultimately achieve the purpose of inducing downstream gene transcription, thus affecting the growth, differentiation and reproduction of cells.

\section{Treatment of primary liver cancer}

In the clinical treatment, the common treatment methods include transcatheter arterial chemoembolization, [2], percutaneous ethanol injection, radiofrequency ablation, [4], high-intensity focused ultrasound treatment, etc.. In addition to the commonly used methods, cryotherapy, laser therapy, interventional radiology and gene therapy have been applied in clinical surgery. In the treatment of liver cancer therapy in numerous found early excision method is mainly used in surgery, removal of cancerous regions of liver cells, combined with chemotherapy and radiotherapy, the potential to kill cancer cells in vivo. In the late stage, a variety of comprehensive treatment modalities, such as transcatheter arterial chemoembolization (TACE) and surgical resection, were used to treat hepatic cancer with hepatic arterial chemoembolization. In the field of basic research, related research group found that drug eluting safety, has high clinical application of Pearl embolism therapy 
in the treatment of advanced hepatocellular carcinoma in the curative effect, low complication rate, and the recent clinical curative effect of transcatheter arterial chemoembolization with [11]. But whether it's early or late, the patient has to suffer from intense chemotherapy pain and side effects of the drug. On primary liver cancer is the clinical treatment situation, primary liver cancer is still a lack of "special treatment" method, various methods of clinical application is palliative treatment, therefore, for primary liver cancer "special treatment" means urgent.

\section{References}

[1].Lu, Z, Sun, W, Wen, F, et, Clinical, application, of, percutaneous, drainage, in, treating, hepatocellular, carcinoma, with, bile, al., duct, tumor, thrombus[J]., Contemporary, Oncology, 2013, 17

[2].Liu Yang, Wu Jinming. Advances in the study of molecular mechanisms of primary liver cancer [J]. medical information, 2009, 19 (2): 109-112.

[3].Li Xinghua, Xu Yunhong, Xu Aifang, et al. Abnormal expression of GST-P and mutation of p53 gene in LEC rats with spontaneous liver cancer [J]. Journal of Second Military Medical University, 2005, 26 (1): 113-115.

[4]. Li Qiang. Clinical therapy of liver cancer [J]. 2010.

[5]. Chen planning. Liver tumor surgery, [M]. people's Military Medical Publishing House, 2011.

[6]. Xie Xie Feng, Yang Jiamei. Current study on the pathogenesis and prevention of primary liver cancer [J]. Chinese medicine, 2009 (3): 170-173.

[7]. Li Li, Yao Shukun, Ma Ying, et al. The role of TGF beta 1/Smads signal transduction pathway in the pathogenesis of primary liver cancer [J]. Clinical Hepatology, 2007, 23 (4): 270-273.

[8]. Deng Jinghuan, Qin Xue. Advances in the study of the pathogenesis of primary liver cancer [J]. Journal of environment and health, 2007, 24 (11): 924-926.

[9].Hong, C, W, Libutti, S, K, Wood, B, J., Liposomal, doxorubicin, plus, radiofrequency, ablation, for, complete, necrosis, of, a, hepatocellular, carcinoma.[J].,, Current, Oncology, 2013, (e274.)

[10]. Salem R, Gilbertsen M, Butt Z, et al. Increased quality of life among hepatocellular carcinoma patients treated with radioembolization, compared with chemoembolization.[J]. Clinical Gastroenterology the Official Clinical Practice \& Hepatology Journal of the American Gastroenterological Association, 2013, 11 (10): 1358-1365.

[11]. Wang Jianguo. Advances in transcatheter arterial chemoembolization for hepatocellular carcinoma [J]. Chinese and foreign health abstracts, 2010, 07 (24): 95-96. 